\title{
Endovascular Thrombectomy for Acute Ischemic Stroke in the Filipino Population: A Clinical Experience From a Single Tertiary Center in Metro Manila, Philippines
}

Glenn Anthony Constantino ( $\square$ gaconstantinomd@gmail.com )

The Medical City, Philippines

Miguela Marie Senga

The Medical City, Philippines

Jo Ann Soliven

The Medical City, Philippines

Victor Erwin Jocson

The Medical City, Philippines

\section{Research Article}

Keywords: Thrombectomy, Endovascular procedures, Ischemic stroke, Treatment outcomes

Posted Date: December 8th, 2021

DOI: https://doi.org/10.21203/rs.3.rs-1057515/v1

License: (c) (1) This work is licensed under a Creative Commons Attribution 4.0 International License.

Read Full License 


\section{Abstract \\ Background and Purpose}

Throughout the years, stroke has remained one of the primary causes of significant morbidity and mortality. Among the therapeutic options for acute stroke management, endovascular thrombectomy intended to remove the thrombi within the intracerebral vasculature and restore adequate perfusion to the surrounding penumbra. It was recommended for eligible patients who were within 6-24 hours after the onset of neurologic symptoms. In the Philippines, only a few tertiary healthcare institutions were able to offer and perform endovascular thrombectomies. The aim was to describe the profile and discharge outcomes of endovascular thrombectomy for acute ischemic stroke at a tertiary hospital in our country.

\section{Methodology:}

In this retrospective study, 924 patients were admitted for acute ischemic stroke from October 2018 August 2021. However, only 31 patients underwent mechanical thrombectomy and their records were thoroughly reviewed. Clinical and functional outcomes were measured using the National Institutes of Health Stroke Scale (NIHSS) and Modified Rankin Score (MRS), respectively.

\section{Results}

Among the patients included in the study, 29 subjects (93.5\%) had moderate to severe disability (MRS 35 ) and 17 (54.8\%) had moderate stroke (NIHSS 5-15) on admission. The identified site of the cerebrovascular thrombi was within the M1 segment of the middle cerebral artery $(41.9 \%, n=13)$. The stent retriever approach was performed in 19 of the subjects (61.2\%). Upon discharge, only 17 (22.6\%) had favorable functional outcomes (MRS 0-2) and 6 (19.3\%) resulted in mortality.

\section{Conclusion}

Overall, endovascular thrombectomy is a promising treatment strategy for large vessel acute ischemic stroke in a developing country.

\section{Introduction}

Acute ischemic stroke remains to be a neurologic emergency that leads to significant mortality and morbidity worldwide, including Asia ${ }^{1}$. Asia, where many of its countries are developing economies, holds $60 \%$ of the world's population and carries some of the highest stroke disease burden. Although epidemiological data on stroke incidence is available for the countries in East Asia, there remains paucity of data in the South East region of the continent ${ }^{2}$. More specifically in the Philippines, epidemiological 
studies have been undertaken including nationwide, community-based, and hospital-based studies. All of which did not have reliable data with regard to stroke incidence and overall burden in the country ${ }^{3}$. In addition to this, much less data have been published with regard to acute ischemic stroke management and therapies for recanalization in the country.

In 1996, the National Institute of Neurological Disorders and Stroke (NINDS) trial utilized intravenous recombinant tissue plasminogen activator (IV-rTPA) to reduce or reverse the extent of neurologic injury from acute ischemic stroke if given within 3 hours after symptom onset. Following this, additional trials have demonstrated favorable outcomes and benefit when the time window for the administration of IVrTPA was extended up to 4.5 hours from symptom onset. In 1999, the Philippines approved the use of IVrTPA but its utility in the clinical setting remained to be reserved due to its prohibitive cost ${ }^{4}$. A multicenter retrospective cohort study by Navarro et al in 2018 showed a thrombolysis rate of $1.4 \%$ with only 157 from a total of 11, 874 patients receiving IV-rTPA from 2014-2016 ${ }^{4}$.

Alternatively, in 2015, the Multicenter Randomized Clinical Trial of Endovascular Treatment for Acute Ischemic Stroke in the Netherlands (MR CLEAN) presented the results of their study comparing the safety and efficacy of intra-arterial therapies which were broadly divided into clot retrieval or mechanical thrombectomy versus clot dissolution with the use of a thrombolytic agent (IV-rTPA) for acute ischemic stroke within 6 hours of neurologic symptom onset. They determined that endovascular thrombectomy in the setting of anterior circulation large vessel occlusion was safe and effective which led to favorable outcomes post-intervention such as functional independence, smaller final infarct volume, and good reperfusion ${ }^{5}$. The synthesis of these results, along with other numerous trials, supported the role of stent retriever thrombectomy as a standard treatment in a carefully defined population. However, in our local setting, there has not been any data documenting the utilization and outcomes of patients who have undergone endovascular thrombectomy. This is, in part, due to the cost of treatment, and availability of appropriate equipment and resources including the number of trained endovascular interventionalists. This study aims to describe the demographic and clinical profiles and discharge outcomes of patients to whom endovascular thrombectomy for acute ischemic stroke was rendered in a tertiary hospital in the Philippines.

\section{Methodology}

This was a retrospective descriptive study of patients who underwent endovascular thrombectomy that was performed at a single tertiary center in the Philippines from September 2018 to August 2021. This was submitted to the Institutional Review Board of the hospital and gained ethical approval for the study. The study solely utilized medical records review for data collection hence the hospital Institutional Review Board allowed the informed consent to be waived for this study. Eligible patients were 18 to 90 years old, clinically assessed to have acute ischemic stroke, and with radiographic evidence of large vessel occlusion as seen either on magnetic resonance angiography (MRA) or computed tomographic angiography (CTA), had National Institutes of Health Stroke Scale score of 4 or greater (range 0 [no symptoms] to 42 [most severe neurologic condition]), was pre-morbidly functionally independent, those 
who fulfilled the criteria for administration of IV-rTPA, and those within the first 24 hours from neurologic symptom onset. The NIHSS was assessed at baseline, 24 hours post-intervention, and upon discharge. The Modified Rankin Score (mRS) was assessed at baseline and upon discharge. Excluded in the study were those who had radiographic evidence of any intracranial hemorrhage and those who did not have any occlusion on either MRA or CTA. In some of the patients, intravenous rTPA was administered prior to thrombectomy. With this, the indications and contraindications for IV-rTPA were observed as detailed in the NINDS trial. The decision in regards to the neuroimaging (CT or MRI) and dose of IV-rTPA, whether $0.9 \mathrm{mg} / \mathrm{kg}$ body weight or $0.6 \mathrm{mg} / \mathrm{kg}$ body weight, was left to the discretion of the attending neurologist. For either dose, $10 \%$ of the computed volume was administered for 1 minute as intravenous bolus with the remainder volume infused over 60 minutes.

The baseline demographic characteristics of the patients were gathered such as age, sex, and co-morbid medical conditions considered being vascular risk factors. These vascular risk factors were defined as, but were not limited to, arterial hypertension (systolic blood pressure or SBP $\geq 140 \mathrm{mmHg}$, and/or diastolic blood pressure or $\mathrm{DBP} \geq 90 \mathrm{mmHg}$ on repeated examination or a patient on any antihypertensive medication), diabetes mellitus (fasting plasma glucose $[\mathrm{FPG}] \geq 126 \mathrm{mg} / \mathrm{dL}[7.0 \mathrm{mmol} / \mathrm{L}]$, $\mathrm{HbA} 1 \mathrm{C} \geq 6.5 \%[48 \mathrm{mmol} / \mathrm{mol}]$, or a patient on insulin therapy or any oral hypoglycemic medication), atrial fibrillation (previously or newly documented on EKG or 24 hour Holter monitoring), dyslipidemia (a patient on statin therapy or any lipid-lowering medication, or with elevated triglyceride and or low-density lipoprotein[LDL] levels), with previous history of cerebrovascular disease or coronary artery disease including myocardial infarction, valvular heart disease, congestive heart failure (whether with preserved $[\geq 40 \%]$ or reduced $[<40 \%]$ ejection fraction), bronchial asthma or chronic obstructive pulmonary disease, chronic kidney disease, and significant smoking history. Additionally, baseline capillary blood glucose (CBG), systolic and diastolic blood pressure levels upon arrival at the hospital, intake of either antiplatelet or anticoagulant, initial neuroimaging modality used, and administration of IV-rTPA prior to thrombectomy was included as baseline demographic information.

The primary outcomes that were measured included the clinical status and functional independence using the National Institutes of Health Stroke Scale (NIHSS) and Modified Rankin Score (mRS, range from 0[no symptoms] to 6[death]), respectively. The NIHSS score were assessed at baseline, 24 hours after the procedure, and upon discharge. Stroke severity was categorized as mild (NIHSS 1-4), moderate (NIHSS 515), moderate to severe (NIHSS 16-20), and severe (NIHSS 17-41). Similarly, functional independence was also assessed prior to onset of stroke, upon admission, and upon discharge. The mRS was dichotomized as follows: favorable/independent, mRS 0-2 and unfavorable/dependent mRS 3-5. Additional outcome measures were common sites of occlusion as documented on cerebral angiography, and timeline from stroke to onset to groin puncture and recanalization. The primary endovascular approach - use of stent retriever, aspiration or suction embolectomy, combined stent retriever and aspiration technique, or deployment of intravascular stent - and number of stent passes were also documented. Furthermore, the modified Thrombolysis In Cerebral Infarction ( $\mathrm{mTICl}$ ) was used to determine the degree of reperfusion, with $\mathrm{mTICI} 2 \mathrm{~B}$ or 3 regarded as successful reperfusion. Finally, hemorrhagic transformation was determined using non-contrast CT scan (NCCT) 24 hours after thrombectomy and was categorized using 
the radiologic classification that arose from European Cooperative Acute Stroke Study (ECASS). Lastly, the post-thrombectomy hemorrhage was classified as either asymptomatic or symptomatic.

Symptomatic intracerebral hemorrhage ( $\mathrm{SICH}$ ) was defined by Safe Implementation of Thrombolysis in Stroke-Monitoring Study (SITS-MOST) as [1] of parenchymal hemorrhage type 2 as defined by ECASS seen on neuroimaging 22 to 36 hours after intervention or earlier if the imaging was done due to clinical and neurologic deterioration, [2] increase in the NIHSS $\geq 4$ from baseline or from the lowest NIHSS score between baseline, [3] or resultant to mortality within 24 hours. $^{13}$

\section{Results}

A total of 31 patients out of 924 acute ischemic stroke patients qualified and underwent endovascular thrombectomy from September 2018 until August 2021. Endovascular thrombectomy was first done at our institution in September 2018 and since then had a steady increase in the number of procedures done per year. The mean age was $64(S D \pm 12)$ and there were more males than females (17:14). About half of the patients had hypertension, and atrial fibrillation. Less frequently, patients had diabetes mellitus, previous history of stroke, coronary heart disease, and valvular heart disease. More than half of the patients were smokers or had significant previous smoking history. The median (interquartile range) capillary blood glucose on hospital arrival was 8 (7-9), systolic blood pressure was 140 (125-170), and diastolic blood pressure (80 (70-90). Among the patients, 16 (52\%) were taking either antiplatelets (Aspirin, Clopidogrel) or anticoagulants (Warfarin, Dabigatran, Apixaban, Rivaroxaban) prior to stroke onset (Table 1). The primary neuroimaging modality utilized in this study was computed tomography with angiography (CT/CTA) accounting for $64.5 \%$ of the population and the remainder $(35.5 \%)$ used noncontrast magnetic resonance imaging with angiography (MRI/MRA). A total of 11 patients (35.5\%) received intravenous rTPA. Ten patients were given a dose of $0.9 \mathrm{mg} / \mathrm{kg}$ body weight, and 1 patient received a lower dose at $0.6 \mathrm{mg} / \mathrm{kg}$ body weight. 


\section{Demographic Profile}

Age (mean $\pm S D)$

$64.0 \pm 11.8$

Gender (M:F Ratio)

$17: 14$

Vascular Risk Factors

$\mathrm{n}=31(\%)$

Smoking

$18(58)$

Hypertension

$17(54.8)$

Atrial Fibrillation

$17(54.8)$

Diabetes Mellitus

$12(38.7)$

History of Cerebrovascular Disease (CVD)

$5(16.1)$

Valvular Heart Disease

$4(12.9)$

Coronary Artery Disease (CAD) and/or history of Acute Coronary Syndrome (ACS)

$44(12.9)$

Dyslipidemia

$2(6.5)$

Chronic Kidney Disease

$2(6.5)$

Bronchial Asthma and/or Chronic Obstructive Pulmonary Disease (COPD)

$2(6.5)$

Others:

$1(3.2)$

Benign Prostatic Hyperplasia

1 (3.2)

Pulmonary Tuberculosis

1 (3.2)

Hyperuricemia

Liver cirrhosis

Malignancy

Pre-stroke antithrombotic agents

Intake of antiplatelet

9 (29)

Intake of anticoagulant

7 (22.6)

Capillary Blood Glucose on hospital arrival, $\mathrm{mg} / \mathrm{dL}$, median (interquartile range)

$8(7-9)$

Systolic Blood Pressure on hospital arrival, $\mathrm{mmHg}$, median (interquartile range)

$140(125-170)$

Diastolic Blood Pressure on hospital arrival, $\mathrm{mnmHg}$, median (interquartile range)

Imaging Modality

$80(70-90)$

CT with Angiography $\mathrm{n}=31(\%)$ $20(64.5)$ 


\section{Demographic Profile}

Non-contrast MRI with Angiography

$11(35.5)$

Table 2 shows the clinical and functional outcomes measure in this study. The NIHSS score was used to determine clinical improvement and was assessed at multiple and different timelines (baseline [prethrombectomy], 24 hours post-thrombectomy, and upon discharge) during each patient's course. The median (IQR) NIHSS at baseline was 14 (11-19), at 24 hours post-thrombectomy was also 15 (9-21) [pvalue $=1]$ ), and at discharge was 8(2-10) [p-value $<0.00001]$. Consequently, functional capacity (mRS) was also assessed at different timelines (baseline [pre-morbid], pre-thrombectomy [upon admission], and at discharge). Results were dichotomized between favorable outcome/independent (mRS 0-2), and unfavorable outcome/dependent (mRS 3-5). Pre-mobidly, majority (93.5\%) had mRS of $0-2$ and the remainder had mRS 3-5 as sequelae of previous stroke. Subsequently, upon onset of neurologic deficits and on admission, $29(93.5 \%)$ of the patients had mRS of 3-5, while only $2(6.5 \%)$ remained to have mRS of $0-2$. On discharge, 17 (22.6\%) were able to regain good functional capacity while 15 patients (48.4\%) maintained functional dependence, and a total of 9 patients $(29.0 \%)$ resulted to in-hospital mortality. It is important to note, however, that among those who resulted to mortalities 3 of them were due to complications of chronic care and prolonged hospitalization and were not direct consequences of endovascular thrombectomy. 
Table 2

Clinical and Functional Outcomes

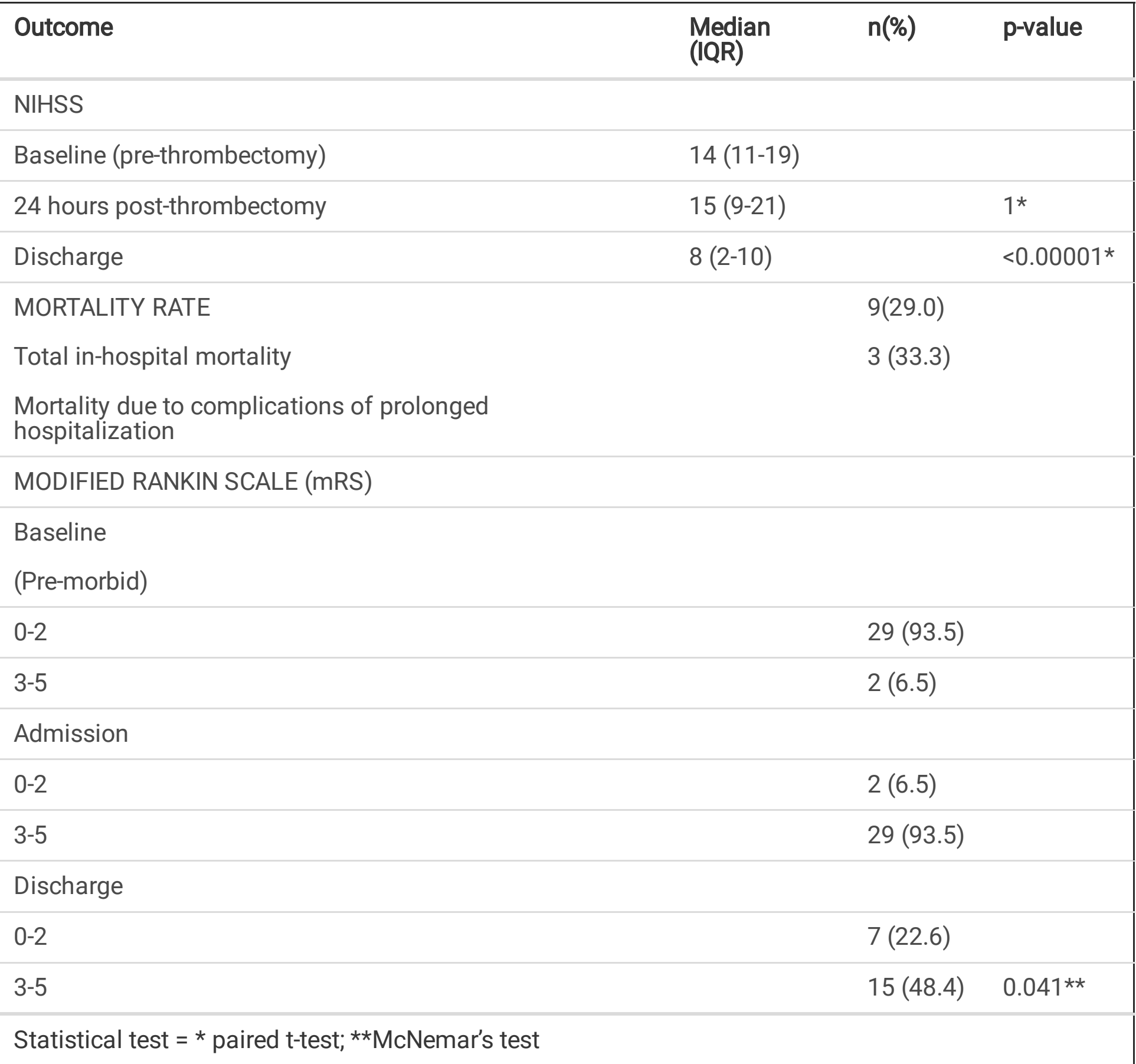

Procedural characteristics were also determined such as occlusion site as seen on cerebral angiography, timelines from symptom onset to groin puncture and recanalization, endovascular technique, number of stent passes, and degree of reperfusion (Table 3). The only post-procedure complication included was the evidence of hemorrhagic transformation as documented by non-contrast CT at any time within 24 hours after the thrombectomy. The major sites of endovascular thrombi were the proximal segments of the middle cerebral artery (MCA). Specifically, $41.9 \%$ of the occlusion was noted within the M1 segment and $12.9 \%$ was seen within the M2 segment of the MCA. Furthermore, $11(35.5 \%)$ of the patients had occlusion in their intracranial internal carotid artery (ICA). Uncommon sites of occlusion involved the 
distal M3 segment of the MCA, as well as the posterior circulation particularly the P1 segment of the posterior cerebral artery (PCA), and the basilar trunk. The mean ictus to groin time was 6.7 hours $(S D \pm 2.2)$, and the mean ictus to recanalization time was 7.8 hours $(S D \pm 1.9)$. Among the 31-endovascular procedures that were performed, 3 procedures were terminated intra-operatively due to arduous vascular access and futility of attempts. The intra-operative findings in these aborted procedures were occlusive thrombus at the $\mathrm{C} 7$ segment of the ICA, partially occluded basilar trunk, and calcified thrombosis at the post bifurcation braches of the M2 segment of the MCA. All of which precluded the adequate passage of the vascular microcatheters and stent retrievers. Moreover, 2 of the procedures resulted instead to intraoperative intra-arterial administration of rTPA as there was no angiographic evidence of a large vessel occlusion. Overall, only 26 out of the 31 procedures went on to complete the endovascular thrombectomy. The primary techniques used for endovascular thrombectomy were stent retriever approach (61.3\%), aspiration or suction embolectomy (9.7\%), and a combined stent retriever and aspiration technique (12.9\%). Single passage of the stent retriever was achieved in $14(60.9 \%)$ of the 23 patients to whom stent retriever or combined stent retriever/aspiration approach was used. Successful post-thrombectomy reperfusion seen on final angiography was achieved in 24 (92.3\%) of the patients. Finally, intracranial hemorrhage was seen in 9 of the patients following endovascular intervention, and 1 did not have postprocedure neuroimaging. Using the ECASS radiologic classification for hemorrhagic transformation, a little over half of the patients with intracerebral hemorrhage had massive bleeding ( $\mathrm{PH} 1$ and $\mathrm{PH} 2)$ following the endovascular intervention. Among those who had post-thrombectomy hemorrhage, 5 (55.6\%) had symptomatic $\mathrm{ICH}(\mathrm{sICH})$ and 4 of the patients (44.4\%) were asymptomatic. 
Table 3

Procedural Characteristics

$n(\%)$

Mean(SD)

Timeline from Ictus

Groin Puncture (hours)

$6.7 \pm 2.2$

Recanalization (hours)

$7.8 \pm 1.9$

Site of occlusion $(n=31)$

Internal Carotid Artery (ICA)

$11(35.5)$

Middle Cerebral Artery (MCA)

18 (58.1)

M1

$13(41.9)$

M2

$4(12.9)$

M3

1 (3.2)

Posterior Cerebral Artery (PCA)

1 (3.2)

Basilar artery (BA)

1 (3.2)

Endovascular approach $(n=31)$

Stent Retriever

19 (61.3)

Aspiration/Suction Embolectomy

$3(9.7)$

Combined Stent Retriever and Aspiration Embolectomy

$4(12.9)$

Intra-arterial rTPA

$2(6.5)$

Terminated Procedure

$3(9.7)$

Number of passes $(n=23)$

1 pass

$14(60.9)$

$>1$ pass

$9(39.1)$

$\mathrm{mTICI}(\mathrm{n}=26)$

$2 b-3$

$24(92.3)$

$1-2 a$

2 (7.7)

No post-thrombectomy hemorrhage

$21(67.7)$

With post-thrombectomy hemorrhage $(n=9)$

$9(29.0)$

mTICl - Modified Thrombolysis In Cerebral Infarction HI - Hemorrhagic Infarct PH - Parenchymal Hemorrhage 


\begin{tabular}{|c|c|c|}
\hline & $\mathrm{n}(\%)$ & Mean(SD) \\
\hline HI 1 & \multicolumn{2}{|l|}{$2(22.2)$} \\
\hline HI 2 & \multicolumn{2}{|l|}{$2(22.2)$} \\
\hline $\mathrm{PH} 1$ & \multicolumn{2}{|l|}{$4(44.4)$} \\
\hline $\mathrm{PH} 2$ & \multicolumn{2}{|l|}{$1(11.1)$} \\
\hline Symptomatic Intracerebral Hemorrhage (sICH) & \multicolumn{2}{|l|}{$5(55.6)$} \\
\hline Asymptomatic Intracerebral Hemorrhage & \multicolumn{2}{|l|}{$4(44.4)$} \\
\hline No neuroimaging done after thrombectomy & \multicolumn{2}{|l|}{$1(3.2)$} \\
\hline $\begin{array}{l}\text { mTICI - Modified Thrombolysis In Cerebral Infa } \\
\text { Hemorrhage }\end{array}$ & Infarct $F$ & arenchymal \\
\hline
\end{tabular}

\section{Discussion}

This study showed the demographic profile and clinical features of the 31 patients who underwent endovascular thrombectomy at a single tertiary center in the Philippines. The procedural characteristics of endovascular thrombectomy were likewise described. Majority of the population were males with a mean age of 64 years. The common risk factors were smoking, hypertension, and atrial fibrillation. Intake of antithrombotic agents prior to stroke was noted in 16 (51.6\%) patients. The primary neuroimaging modality of choice was CT with angiography (64.5\%). Intravenous rTPA was administered in 11 patients (35.5\%) prior to the endovascular intervention. The mean NIHSS was 15 and 7 at baseline and upon discharge, respectively. The differences between the NIHSS scores and MRS from admission to discharge were statistically significant. The primary endovascular approach used was the stent retriever technique $(61.3 \%)$ and majority was successful with a single stent pass. The common sites of thrombi occlusion were noted within the proximal segments of the middle cerebral artery $(58.1 \%)$ and the intracranial segments of the internal carotid artery (35.5\%). The mean time from onset of neurologic symptoms to groin puncture was 6.7 hours and recanalization was achieved at a mean time of 7.8 hours from stroke onset. Successful reperfusion ( $\mathrm{mTICl} 2 \mathrm{~b}-3)$ was seen in $24(92.3 \%)$ of the patients and $5(16.1 \%)$ had severe post-thrombectomy intracranial hemorrhage. The in-hospital mortality rate was $9(29 \%)$ and functional dependence upon discharge was seen in 15 (48.4\%) of the patients.

Endovascular thrombectomy (EVT) has become a standard intervention in acute ischemic stroke patients with large vessel occlusion within 6 hours from symptom onset following the publication of 5 randomized clinical trials back in $2015^{5-9}$. In Asia, there is still limited data in the utilization of endovascular thrombectomy in the management of acute ischemic stroke patients. Tsang et al reviewed the accessibility gap of endovascular thrombectomy for acute ischemic stroke in Asia. They gathered data from 42 respondents representing 19 countries, and 35 cities. It was revealed that there was large disproportion in the accessibility of EVT in Asia. High-income countries had undoubtedly better access to 
both EVT and intravenous thrombolysis (IVT) in comparison to middle- and low-income countries. The Philippines was one of the countries - together with Bangladesh, Mongolia, Nepal, and Pakistan - who had limited access to both EVT and IVT despite a high stroke disease burden. The primary impediments that were identified as contributory to the underwhelming EVT practice were lack of a unified territorywide triage system, insufficient comprehensive stroke hospitals, and inadequate number of neurointerventionists who will perform the procedures. Additionally, inadequate awareness and education of both the physicians and the patients with regard to this therapy as well as the prohibitive cost of treatment and the device were also identified as common issues. This is of major concern for the developing countries where patients shoulder the burden of cost as out-of-pocket ${ }^{10}$. $\mathrm{Ng}$ et al conducted a pilot survey reviewing the state of acute stroke care specific to the Southeast Asia (SEA) region. They identified the status of endovascular thrombectomy in Southeast Asian countries. Specifically, they determined the availability of acute stroke thrombectomy devices, number of EVT sites and interventional neuroradiologists (INR), number of EVT procedures in each Southeast Asian country, and the projected growth of in number of EVT cases. Stentrievers were introduced in majority of the SEA countries in 2016 only after the publication of successful EVT trials in 2015. They found that EVT service is not available 4 of 10 SEA countries, namely Myanmar, Laos, Cambodia, and Brunei. Among the countries that offer EVT, majority of the sites were located in city centers and not all offer a round-the-clock service. Moreover, a peculiar situation was noted in the Philippines, as there was disparity between the number of EVT sites and number of active INRs. This reflected the under-utilization of available resources ${ }^{11}$. As such, our institution only documented its first EVT procedure only in 2018.

Though there was a lack of published data on EVT in the SEA, China was able to provide a view of EVT utility in their country. Yang et al, in 2019, conducted a trial at 41 academic tertiary centers in China in order to evaluate the efficacy and safety of endovascular thrombectomy with or without intravenous rTPA. They screened 1586 patients and were able to enroll 656 patients who were then randomized into 2 groups, thrombectomy-alone and combination-therapy groups. The primary outcome they measured was functional capacity at 90 days post intervention using the modified Rankin Scale score (mRS). Their results showed that endovascular thrombectomy alone was non-inferior to combination therapy with IVrTPA $^{12}$.

Comparing our experience with the large clinical trials, endovascular thrombectomy in the Philippines is still greatly under-utilized. It is important to note, however, that to the best of our knowledge this study showed the most number of endovascular thrombectomies performed in the Philippines. This mirrors the previously identified gap between accessibility and utility of endovascular thrombectomy. Our study was designed to demonstrate the clinical and functional outcomes of endovascular thrombectomy among Filipinos in order to increase awareness with regard to this technology among the Filipino patients and physicians. Our results showed that the extent of neurologic deficit was significantly reduced and the functional outcomes were improved after endovascular thrombectomy.

Our study has limitations. First, it was a retrospective study and the data collected were mainly from review of medical records. Second, the sample population was significantly smaller in comparison to the 
clinical trials. Third, the outcomes measured in this study did not include the standard 90-day follow-up limiting an accurate assessment of efficacy. Lastly, this study documents the experience of only a single center in the entire country. Despite these limitations, we are optimistic that this study will initiate a cascade of similar inquiry that will augment our local data and knowledge to this therapy and hopefully embolden Filipino physicians to offer and utilize endovascular thrombectomy to Filipino patients.

\section{Declarations}

\section{Ethics approval and consent to participate}

The study protocol for this research was reviewed and approved by the Institutional Review Board of The Medical City hospital. The Institutional Review Board of The Medical City hospital allowed the informed consent to be waived for this study since this was a retrospective study, which utilized medical record review for data collection. All methods of the study were carried out in accordance with relevant guidelines and regulations.

\section{Consent for publication}

Not applicable.

\section{Availability of data and materials}

All data generated or analyzed during this study are included in this article (and its supplementary information files).

\section{Competing interests}

The authors of this study have nothing to disclose. The authors do not have any financial, commercial, legal, or professional relationship with organizations that can affect or influence the results of this research.

\section{Funding}

This research did not receive any form of funding from any sponsor.

\section{Author's contributions}

All authors equally contributed to the development and writing of this study.

\section{Acknowledgements}

Not applicable.

\section{References}


1. Turana, Y, Tengkawan, J, Chia, YC, et al; the HOPE Asia Network. Hypertension and stroke in Asia: A comprehensive review from HOPE Asia. J Clin Hypertens. 2021; 23: 513-521. https://doi.org/10.1111/jch.14099

2. Venketasubramanian, N., Yoon, B. W., Pandian, J., \& Navarro, J. C. (2017). Stroke Epidemiology in South, East, and South-East Asia: A Review. Journal of stroke, 19(3), 286-294. https://doi.org/10.5853/jos.2017.00234

3. Navarro J, C, Venketasubramanian N: Stroke Burden and Services in the Philippines. Cerebrovasc Dis Extra 2021;11:52-54. doi: 10.1159/000516238

4. Navarro JC, San Jose MC, Collantes E, Macrohon-Valdez MC, Roxas A, Hiyadan J, et al. Stroke Thrombolysis in the Philippines. Neurol Asia. 2018;23(2):115-20

5. Berkhemer OA, Fransen PS, Beumer D, et al. A randomized trial of intraarterial treatment for acute ischemic stroke. N Engl J Med. 2015;372:11-20.

6. Saver JL, Goyal M, Bonafe A, et al. Stent-retriever thrombectomy after intravenous t-PA vs. t-PA alone in stroke. N Engl J Med 2015;372:2285-2295.

7. Goyal M, Demchuk AM, Menon BK, et al. Randomized assessment of rapid endovascular treatment of ischemic stroke. N Engl J Med 2015;372:1019-1030.

8. Campbell BCV, Mitchell PJ, Kleinig TJ, et al. Endovascular therapy for ischemic stroke with perfusionimaging selection. N Engl J Med 2015;372:1009-1018.

9. Jovin TG, Chamorro A, Cobo E, et al. Thrombectomy within 8 hours after symptom onset in ischemic stroke. N Engl J Med 2015;372:2296-2306.

10. Tsang, Anderson Chun On; Yang, I-Hsiao; Orru, Emanuele; Nguyen, Quang-Anh; Pamatmat, Roselyn V; Medhi, Gorky; Wan, Yue; Huang, Song (2019). Overview of endovascular thrombectomy accessibility gap for acute ischemic stroke in Asia: A multi-national survey. International Journal of Stroke, 0, 174749301988134-. doi:10.1177/1747493019881345

11. Ng, Justin Christopher; Churojana, Anchalee; Pongpech, Sirintara; Vu, Luu Dang; Sadikin, Cindy; Mahadevan, Jeyaledchumy; Subramaniam, Josephine; Jocson, Victor Erwin; Lee, Wickly (2018). Current state of acute stroke care in Southeast Asian countries. Interventional Neuroradiology, (), 159101991881180-. doi:10.1177/1591019918811804

12. Yang $P$, Zhang $Y$, Zhang $L$, et al. Endovascular Thrombectomy with or without Intravenous Alteplase in Acute Stroke. N Engl J Med. 2020 May 21;382(21):1981-1993. doi: 10.1056/NEJMoa2001123. Epub 2020 May 6. PMID: 32374959.

13. Wahlgren, Nils \& Ahmed, Niaz \& Dávalos, Antoni \& Ford, Gary \& Grond, Martin \&Hacke, Werner \& Hennerici, Michael \& Kaste, Markku \& Kuelkens, Sonja \& Larrue, Vincent\& Lees, Kennedy \& Roine, Risto \& Soinne, Lauri \& Toni, Danilo \& Vanhooren, Geert.(2007). Thrombolysis with alteplase for acute ischaemic stroke in the Safe Implementation of Thrombolysis in Stroke-Monitoring Study (SITS-MOST): an observational study. Lancet.369. 275-82. 10.1016/S0140-6736(07)60149-4.

\section{Supplementary Files}


This is a list of supplementary files associated with this preprint. Click to download.

- EndovascularThrombectomyData.xlsx 